\title{
Comparación de infusión automática respecto a ma- nual en hemodiafiltracion "on line" post - dilucional
}

\author{
Premio Fresenius Medical Care para Enfermería. Hemodiafiltración en línea
}

Ana Vanessa Fernández Martínez - Salvadora Soto Ureña - Maria Arenas Fuentes - Francisco Horrillo Jimenez - Natalia Saez Donaire - Laura Pérez Valencia

Centro de hemodiálisis Nefroclub Cartago. Cartagena

\section{Resumen}

La hemodiafiltración "on-line" postdilucional es el modo de infusión más eficaz para la eliminación de moléculas de diferentes pesos moleculares; también se ha relacionado con un mejor control de la anemia así como del estado nutricional del paciente, confiriendo una menor morbilidad al paciente en hemodiálisis. Recientemente, se han incorporado importantes avances en la tecnología que permiten la prescripción automática del flujo de infusión lo que podría reducir las cargas de enfermería.

El objetivo del presente estudio es evaluar la eficacia de la infusión automática comparada con la manual en la HDF on-line postdilucional, mediante la medición del volumen convectivo final y del aclaramiento de pequeñas moléculas mediante el Kt. También se evalúan las cargas de enfermería medidas por el número de intervenciones relacionadas con la técnica, la valoración de comodidad y eficacia percibida por el personal de enfermería y si hay diferencias en el confort del paciente a lo largo de la sesión.

Se diseña un estudio prospectivo sobre 86 pacientes; tras tres sesiones con cada uno de los modos de reinfusión se aprecian diferencias significati-

\begin{tabular}{|c|} 
Correspondencia: \\
Ana Vanessa Fernández Martínez \\
Centro de Hemodiálisis Nefroclub Cartago \\
C/ Budapest parcela 136- Pol. Ind. Cabezo Beaza \\
30353. Cartagena - Murcia \\
nefroclubcarthago@gmail.com
\end{tabular}

vas tanto en el volumen de sustitución ( 1,5 litros) como en la dosis de diálisis administrada medida mediante $\mathrm{Kt}$ (1,02 litros), que son superiores en la forma automática. Por otra parte, la valoración subjetiva del personal de enfermería es muy superior en el modo automático frente al manual tanto en eficacia percibida como en comodidad. El paciente también expresa un mayor confort en las sesiones con reinfusión automática al disminuir en número de alarmas acústicas y favorecer el descanso. La reinfusión automática aunque con poca repercusión clínica, se muestra como una buena alternativa sobretodo en lo que respecta a la adaptación de la técnica por parte del personal y del propio paciente.

\section{PALABRAS CLAVE:}

- HEMODIAFILTRACION

- CALIDAD DE VIDA

- INFUSION

\section{Comparison of automatic versus manual infu-} sion in post-dilutional online haemodiafiltration

\section{Abstract}

Post-dilutional online haemodiafiltration is the most efficacious infusion method for the elimination of molecules with different molecular weights; it has also been associated to a better control of anaemia and of the nutritional condition of the patient, presenting lower morbility for patients undergoing haemodialysis. Recently, major advances have been made in the technology enabling the 
automatic prescription of the infusion flow, which could reduce the nursing workloads.

The aim of this study is to evaluate the efficacy of automatic infusion compared to manual infusion in post-dilutional online HDF, by measuring the final convective volume and the rinsing volume of small molecules using Kt. The nursing workloads were also evaluated, measured by the number of interventions related to the technique, an evaluation of the convenience and efficacy perceived by nursing staff and whether there were differences in the patient's comfort during the session.

A prospective study was designed of 86 patients; after three sessions with each of the reinfusion modes, significant differences were observed both in the replacement volume ( 1.5 litres) and in the dialysis dose administered, measured using $\mathrm{Kt}$ (1.02 litres), which are higher in the automatic form. Furthermore, the subjective evaluation of nursing staff is much higher in the automatic mode compared to manual, both in perceived efficacy and in convenience. Patients also expressed greater comfort in sessions with automatic reinfusion as the number of acoustic alarms was reduced, favouring rest. Although the clinical effects were slight, automatic reinfusion is shown to be a good alternative especially in relation to the adaptation to the technique by nursing staff and by patients.

\section{KEY WORDS:}

- HAEMODIAFILTRATION

- QUALITY OF LIFE

- INFUSION

\section{Introducción}

En los últimos años se han descrito diferentes técnicas de hemodiafiltración (HDF) con altos volúmenes convectivos, las cuales constituyen una nueva e interesante aproximación a la forma de depuración del riñón nativo ${ }^{1}$. De entre ellas, la HDF on-line postdilucional es el modo de infusión más eficaz para la eliminación de moléculas de diferentes pesos moleculares $^{2}$. La hemodiafiltración on line se ha relacionado con un mejor control de la anemia y una mejora en el estado nutricional del paciente. Del mismo modo, se ha constatado una mayor estabilidad hemodinámica. Todo ello confiere una menor morbilidad al paciente en hemodiálisis ${ }^{3}$. También se ha comunicado por nuestro grupo una mejora en la calidad de vida, medida por el cuestionario SF-36 y el índice de Karnofsky, así como una buena aceptación y valoración por parte del personal de enfermería ${ }^{4}$.

Aunque un subanálisis del estudio observacional DOPPS confiere un descenso de la mortalidad a los tratados con HDF on-line frente a la hemodiálisis convencional de un $23 \%$, este efecto desaparece cuando se ajusta para la misma dosis de diálisis ${ }^{5}$. Por otra parte, resultados del registro de Lombardía muestran una tendencia hacia una mayor supervivencia en las técnicas con alto trasporte convectivo ${ }^{6}$.

Recientemente, se han incorporado importantes avances en la tecnología que permiten la prescripción automática del flujo de infusión (Qi), siempre que se especifiquen los valores de hematocrito y de proteínas totales ${ }^{7}$. Como es obvio, esta automatización podría reducir las cargas de enfermería, habida cuenta que las principales limitaciones de la HDF on-line postdilucional son el aumento de la presión transmembrana (PTM) y la hemoconcentración, que hacen necesaria la atención a las diferentes alarmas, así como el reajuste a la baja del $\mathrm{Qi}^{1}$. Para minimizar este problema, se recomienza establecer un Qi inicial del $25 \%$ del flujo sanguíneo en la reinfusión manual.

El objetivo del presente estudio es evaluar la eficacia de la infusión automática comparada con la manual en la HDF on-line postdilucional, mediante la medición del volumen convectivo final y del aclaramiento de pequeñas moléculas mediante el $\mathrm{Kt}^{8}$. Por otro lado, se evalúan las cargas de enfermería medidas por el número de intervenciones relacionadas con la técnica, la valoración de comodidad y eficacia percibida por el personal de enfermería y si hay diferencias en el confort del paciente a lo largo de la sesión.

\section{Material y Métodos}

Se trata de un estudio prospectivo sobre población prevalente en hemodiálisis de nuestra área de salud. Se incluyeron a los pacientes mayores de 18 años en tratamiento con hemodiafiltración on line posdilucio- 
nal, tras haber sido informados de las características del estudio y dado su consentimiento para él mismo.

En todos los pacientes se realizan 3 sesiones de diálisis (sistema terapéutico 5008 Fresenius Medical Care ${ }^{\circledR}$ ) con sustitución posdilucional, con reinfusión de líquido de sustitución automática, y un Qi inicial según la siguiente fórmula:

Qi $=($ Qb [1- (Hematocrito/100) $\times(1-(7 \times$ proteínas totales/100)] - (tasa de ultrafiltración/60) $\times \mathrm{F}$

Donde:

- Qi es el volumen de reinfusión ( $\mathrm{ml} / \mathrm{min})$

- Qb el flujo sanguíneo ( $\mathrm{ml} / \mathrm{min}$ )

- F el factor de autosustitución en función del dializador

- Hematocrito expresado en \%

- Proteínas totales en mg/dl

- Tasa de ultrafiltración en ml/hora

EI Qi se autoajusta a lo largo de la sesión en función de las cifras de presión transmembrana (PTM). Seguidamente, en cada paciente se procede a realizar 3 sesiones con sustitución posdilucional manual, con un Qi inicial del $25 \%$ del Qb. Si la PTM se eleva por encima de $300 \mathrm{mmHg}$ o se produce hemoconcentración, se reduce el Qi un $15 \%$ con respecto al previo. En todas las sesiones se utiliza el mismo dializador, helixona $1,3-1,6 \mathrm{~m}^{2}$ ). Se recogen los siguientes parámetros de forma horaria y se expresan como valor promedio:

- Litros totales de volumen de sustitución

- Tiempo efectivo de diálisis

- Flujo sanguíneo efectivo (Qb)

- Flujo de líquido dializante (Qd)

- Qi horario (al inicio)

- $\quad$ PTM horaria

- $\mathrm{Kt}$

- Paso a predilución

Se recogen también los siguientes datos: edad, sexo, tiempo y etiología de la insuficiencia renal terminal (IRT). Por otra parte, se realiza una pregunta sobre la eficacia percibida y comodidad por parte del personal de enfermería, y de confort por parte del paciente. Cada pregunta se refiere a una escala del 1 al 5, donde 1 es la valoración mínima y 5 la máxima.

El análisis estadístico se realiza mediante el programa SPSS 13.0 para Windows. Las variables cuantitativas se expresan como media, desviación estándar y rango. Las variables cualitativas, como frecuencia y porcentaje. El contraste de hipótesis para variables cuantitativas se realiza mediante la $\mathrm{t}$-student y la chi-cuadrado de Pearson para variables cualitativas.

\section{Resultados}

Se incluyeron a 86 pacientes cuyas características basales se describen en la tabla 1 . Se aprecian diferencias significativas tanto en el volumen de sustitución $(p=0,005)$, superior en la forma automática $(21,06 \pm 1,76$ litros) que en la manual $(20,55 \pm 1,36$ litros), como $(p<0,001)$ en el $Q i$ promedio de las sesiones $(96,55 \pm 8,59 \mathrm{ml} / \mathrm{min}$ en automática, versus $93,34 \pm 4,44 \mathrm{ml} / \mathrm{min}$ en manual). Los resultados del Qi horario se expresan en la figura 1 , con diferencias significativas en la primera $(p<0,001)$, segunda $(p<0,001)$ y tercera $(p=0,024)$ hora. El $71 \%$ de los pacientes alcanzan 20 litros o más con la autosustitución, frente al $65 \%$ con la sustitución manual $(p<0,001)$.

\begin{tabular}{|c|l|l} 
Edad & & $63,4 \pm 15,3$ \\
\hline & $\begin{array}{c}\text { Hombres } \\
\text { Mujeres }\end{array}$ & $69(80 \%)$ \\
Etiología & $15(20 \%)$ \\
\hline Diabetes & $15(17,4 \%)$ \\
Vascular & $15(17,4 \%)$ \\
Glomerular & $18(20,9 \%)$ \\
Intersticial & $6(7 \%)$ \\
Hereditaria & $11(12,8 \%)$ \\
Desconocida & $19(22,1 \%)$ \\
Otras & $2(2,4 \%)$ \\
\hline Permanencia en diálisis (meses) & $49,9 \pm 44,5$ \\
\hline Proteínas totales (g/dl) & $6,3 \pm 0,7$ \\
\hline Hematocrito & $34,1 \pm 3,8$ \\
\hline
\end{tabular}

Tabla 1. Características basales de los pacientes 


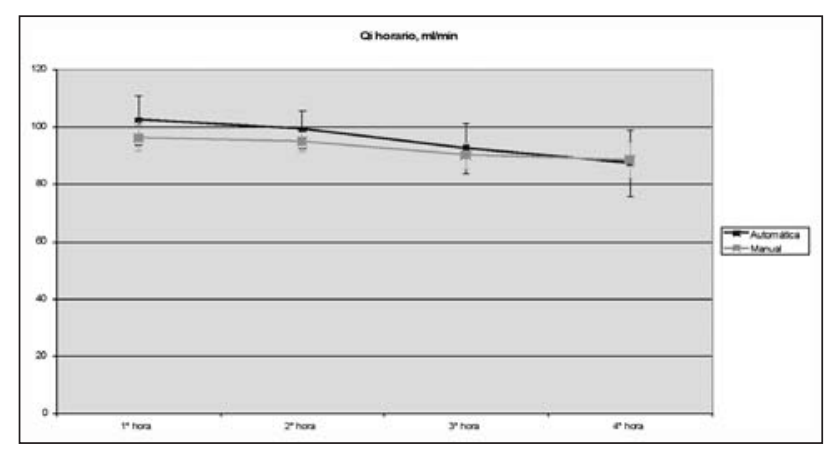

Figura 1. Qi horario.

En cuanto a los resultados de otros parámetros de la diálisis se expresan en la tabla 2. Aunque no existen diferencias significativas en cuanto al Qb y al Qd, la dosis de diálisis administrada medida mediante el Kt es significativamente $(p=0,024)$ mayor en la autosustitución, 1,02 litros en promedio. También se obtiene un mayor tiempo efectivo de diálisis cuando la sustitución es automática con respecto a la manual.

El número de intervenciones del personal de enfermería es muy inferior cuando se emplea la autosustitución, con respecto a la forma manual $(0,29 \pm 0,62$ versus $2,22 \pm 2,22$, respectivamente, $p<0,001)$. Del mismo modo, la valoración subjetiva del personal de enfermería en cuanto a eficacia y comodidad es muy superior en la autosustitución $(p<0,001)$, tal como aparece en la figura 2. Por su parte, el paciente también valora significativamente $(p<0,001)$ la comodidad de la autosustitución $(4,71 \pm 0,35)$ frente a la forma manual $(4,16 \pm 1,18)$.

\begin{tabular}{|c|c|c|c|}
\hline & Automática & Manual & $p$ \\
\hline $\mathrm{Qb}, \mathrm{ml} / \mathrm{min}$ & $384,42 \pm 18,27$ & $382,26 \pm 18,17$ & ns \\
\hline $\mathrm{Qd}, \mathrm{ml} / \mathrm{min}$ & $576,44 \pm 31,15$ & $575,03 \pm 30,65$ & ns \\
\hline PTM, mmHg & $229,10 \pm 23,73$ & $212,91 \pm 28,40$ & $\begin{array}{l}< \\
0,001\end{array}$ \\
\hline $\begin{array}{l}\text { Tiempo } \\
\text { efectivo } \\
\text { (min) }\end{array}$ & $234 \pm 11$ & $231 \pm 9$ & 0,002 \\
\hline Kt & $56,4 \pm 5,22$ & $55,38 \pm 5,72$ & 0,024 \\
\hline
\end{tabular}

Tabla 2. Parámetros de diálisis

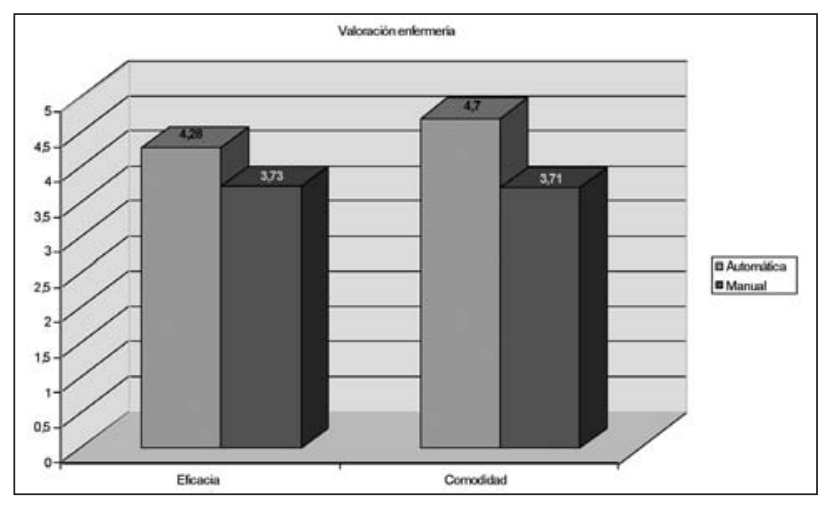

Figura 2. Valoración por parte del personal de enfermería.

\section{Discusión y Conclusiones}

Son múltiples las ventajas descritas de la HDF on line posdilucional; los resultados son buenos incluso a corto y medio plazo ${ }^{9}$, e incluso alguna sociedad científica la considera de elección en su ámbito de aplicación ${ }^{10}$. A la vista de nuestros resultados la infusión automática se muestra más eficaz que la manual tanto en el volumen convectivo final alcanzado como en la dosis de diálisis medida por Kt, si bien en ambos casos (0,49 y 1,02 litros, respectivamente) las diferencias no parecen excesivamente relevantes desde el punto de vista de la práctica clínica. Estos resultados son superponibles a los comunicados por Maduell y $\operatorname{cols}^{11}$, aunque en este estudio la prescripción manual presentaba un mayor volumen convectivo $(1,5$ litros). Esto puede explicarse por las diferencias en el hematocrito (-1\%) y en las proteínas totales $(-0,4$ $\mathrm{g} / \mathrm{dl}$ ), lo que permite una mayor tasa de infusión automática en nuestra serie. Por otro lado, el Qi inicial en el mencionado estudio sobrevalora el $25 \%$ del Qb en $5 \mathrm{ml} / \mathrm{min}$, que en un tiempo medio de 270 minutos puede suponer un volumen añadido de 1,4 litros. Además, la realización de dos sesiones consecutivas puede alterar la tasa de ultrafiltración programada según el día de la semana, cuestión que en nuestro estudio se obvia al analizar de forma conjunta las tres sesiones semanales. Por último, resulta obvio que en nuestra serie podríamos haber sobrepasado el $25 \%$ del flujo sanguíneo efectivo en la infusión manual sin aparentes problemas. Esta pequeña mejora de eficacia se cimienta especialmente en las dos primeras horas de diálisis, tal como queda reflejado en la figura 1, donde la infusión automatizada es claramente superior a la manual en nuestra serie. 
Diferentes estudios relacionan un mayor trasporte convectivo con una tendencia a una mayor supervivencia ${ }^{5-6}$, por lo que de forma habitual, se considera un volumen convectivo óptimo de más de 20 litros, aunque con más de 15 litros puede ser suficiente ${ }^{12}$. En nuestra serie el porcentaje de pacientes que lo alcanzan es un 6\% mayor en la infusión automática. Existe plena coincidencia con el grupo de Maduell en la reducción de las actuaciones de enfermería relacionadas con la técnica en la infusión automática, que en nuestro caso prácticamente desaparecen. No es pues de extrañar la puntuación positiva que expresan en las encuestas de valoración subjetiva I@s enfermer@ del centro, tanto en lo referente a eficacia percibida como a comodidad. Esta impresión se suma a la de la propia técnica de HDF on-line ya reflejada por nuestro grupo 4 .

Finalmente, al paciente le genera una mayor tasa de disconfort la repetición de alarmas acústicas, y por ende, el acercamiento repetido del personal sanitario, en muchas ocasiones interrumpiendo su descanso. La valoración en ese sentido de la infusión automática es excelente.

Por tanto, la infusión automática representa una buena alternativa en la prescripción del flujo de infusión en los pacientes tratados con HDF on-line postdilucional, siempre que se disponga de la tecnología necesaria. La eficacia en volumen de convección y depuración de pequeñas moléculas no es inferior a la que se obtiene con la forma manual, pero con menos problemas de alarmas de PTM por el autoajuste que el monitor realiza de forma automatizada. En algunos pacientes, que lo bordean con la infusión manual, puede ayudar a alcanzar el objetivo de volumen convectivo establecido.

Esto supone una disminución de las intervenciones de enfermería, lo que conlleva una reducción de cargas de trabajo y una buena aceptación y valoración de la técnica, lo que puede ser especialmente importante en aquellos centros que incorporen la HDF online posdilucional a su cartera de servicios. En ese sentido, también es positiva la valoración de mayor confort por parte del paciente.

\section{Bibliografía}

1. Maduell F y Arias M. Indicaciones y prescripción de la hemodiafiltración. Dial Trasp 2008: 29(2): 62-66.

2. Maduell F, García H, Hernández-Jara J y cols. Comparación de la infusión predilucional versus postdilucional en la hemodiafiltración en línea. Nefrología 1998; 18; Supl 3-49

3. Castañeda C, Ciriza A y Díez R. Hemodiafiltración en línea en 52 pacientes: evolución clínica y analítica. Rev Soc Esp Enferm Nefrol 2005; 8(3): 1-6.

4. Alfaro A, Beltrán MI, Gallego B y cols. HDF en línea en nuestros pacientes: calidad de vida y capacidad funcional. Rev Soc Esp Enferm Nefrol 2006; 9 (3) 158-163.

5. Canaud B, Braga-Gresham JL, Marshal MR y cols. Mortality risk for patients receiving haemodiafiltration versus haemodialysis: European results from the DOPPS. Kidney Int. 2006; 69: 20872093.

6. Locatelli F, Marcelli D, Conte F y cols. Comparition of mortality in ESRD patients on convective and difussive extracorporeal treatments. Kidney Int 1999; 55: 286-293.

7. Manual del usuario sistema terapeútico 5008. Fresenius Medical Care.

8. Fernández AV, Soto $S$, Arenas $M$ y cols. Dosis de diálisis medida por dialisancia iónica (Kt). Estudio comparativo con el Kt/V. Factores que influyen en la dosis de diálisis alcanzada. Rev. Soc Esp Nefrol 2009; 12 (2):97-102.

9. Alfaro A, Beltrán MI, Gallego B y cols. HDF en línea en nuestros pacientes: eficacia dialítica y cargas de enfermería. En: Libro de comunicaciones del XXX Congreso de la Sociedad Española de Enfermería Nefrológica; Málaga 12-15 oct, 2005:157-164. 
10. Navarro MJ y Álvarez GM. Técnicas de hemodiálisis. En M: Molina Documento Guía sobre la calidad de la hemodiálisis. Ed Sociedad Murciana de Nefrología. 2008.

11. Maduell F, Arias M, Blasco M y cols. Comparación de infusión automática respecto a la manuales la hemodiafiltración on line postdilucional. Nefrología 2008; 28 (Supl 4): 59.
12. Jirka T, Cesare S, Di Benedetto MP y cols. Mortality risk for patients receiving hemodiafiltration versus hemodiálisis. Kidney Int 2006; 70: 1524. 